\title{
A New Approach for the Solution of Three-Dimensional Magnetohydrodynamic Rotating Flow over a Shrinking Sheet
}

\author{
S. S. Motsa ${ }^{1}$ and S. Shateyi ${ }^{2}$ \\ ${ }^{1}$ Department of Mathematics, University of Swaziland, Private Bag 4, Kwaluseni M201, Swaziland \\ ${ }^{2}$ Department of Mathematics, University of Venda, Private Bag X5050, Thohoyandou 0950, South Africa
}

Correspondence should be addressed to S. Shateyi, stanford.shateyi@univen.ac.za

Received 19 June 2010; Accepted 3 November 2010

Academic Editor: K. Vajravelu

Copyright (c) 2010 S. S. Motsa and S. Shateyi. This is an open access article distributed under the Creative Commons Attribution License, which permits unrestricted use, distribution, and reproduction in any medium, provided the original work is properly cited.

The numerical solution of magnetohydrodynamic (MHD) and rotating flow over a porous shrinking sheet is obtained by the new approach known as spectral homotopy analysis method (SHAM). Using a similarity transformation, the governing equations for the momentum are reduced to a set of ordinary differential equations and are solved by the SHAM approach to determine velocity distributions and shear stress variations for different governing parameters. The SHAM results are analysed and validated against numerical results obtained using MATLAB's built-in bvp4c routine, and good agreement is observed.

\section{Introduction}

The study of flow, heat, and mass transfer problems due to stretching boundary/surface has many applications in technological processes, particularly in polymer systems involving drawing of fibres and films or thin sheets, production of paper, linoleum, roofing shingles, insulting material, and many other applications. In most cases, the polymer sheet is stretched while it is extruded from the dye. The sheet is pulled through viscous liquid with a cooling system to obtain the final product with prescribed characteristics. The moving sheet may introduce a motion in the neighbouring fluid, or alternatively, the fluid may have an independent forced-convection motion which is parallel to that of the sheet. Sakiadis [1] was the first to investigate the flow due to a sheet issuing with constant speed from a slit into a fluid at rest. Since then, many investigators have considered various aspects of this problem and have obtained similarity solutions, and a good amount of references can be found in papers by Crane [2], Magyari and Keller [3-5], Liao and Pop [6], Sparrow, and Abraham [7] and Abraham and Sparrow [8], among others. 
On the other hand, the laminar incompressible boundary layer flow caused by the stretching of a flat surface in rotating fluid has been studied by Wang [9], Rajeswari and Nathi [10] and Nazar et al. [11]. Ariel [12] presented a noniterative numerical scheme which computes the steady, three-dimensional flow of a viscous incompressible fluid past a stretching sheet in single integration. Rashidi and Dinarvand [13] found a totally analytic solution for the problem of condensation or spraying on an inclined rotating disk.

In recent years, problems involving magnetic field have become important. Many metallurgical processes such as drawing, annealing and thinning of copper wire involve the cooling of continuous strips or filaments by drawing them through an ambient fluid. By drawing these filaments in an electrically conducting fluid under the influence of an applied magnetic field, controls the rate of cooling. Kumari and Nath [14] studied, using the homotopy analysis method, the unsteady magnetohydrodynamic viscous fluid and heat transfer of Newtonian fluids induced by an impulsively stretched plane surface in two lateral directions. Fang et al. [15] analytically solved the MHD flow under slip condition over a permeable stretching surface.

The boundary layer flow and heat transfer problem over a moving surface differs from that over a stationary surface caused by the free stream velocity due to the entrainment of the fluid. The moving surface prevents or delays the separation of the boundary layer from the wall by injecting momentum in the existing boundary layer. The magnetic field and the rotation of the fluid increase the surface shear stress for primary flow, but reduce the surface heat transfer. Takhar et al. [16] studied the nonsimilar boundary layer flow of a viscous incompressible electrically conducting fluid over a moving surface in a rotating fluid, in the presence of a magnetic field, Hall currents and the free stream velocity. Vajravelu and Kumar [17] analyzed hydromagnetic flow between two horizontal plates in a rotating system, where the lower is a stretching sheet and the upper is a porous solid sphere.

Literature survey indicates that very little attention has been given to the shrinking flow. Wang [18] developed unsteady shrinking sheet for a specific value of the suction parameter. The rotating flow of an electrically fluid occurs in cosmical and geophysical fluid dynamics. It is also important in the solar cycle and the structure of rotating magnetic stars. Hayat et al. [19] obtained series solution of magnetohydrodynamic and the rotating flow over a porous shrinking sheet using a homotopy analysis method. Sajid and Hayat [20] considered the MHD viscous flow due to a shrinking sheet. The study obtained series solution valid for both two dimensional and axisymmetric shrinking sheet by using homotopy analysis method. Yao and Chen [21] applied the homotopy analysis method to investigate analytically the laminar incompressible viscous flow for a moving semi-infinite flat, or a flat plate continuously shrinking into a slot in a stationary fluid with mass transfer governed by the Blasius equation.

Noor et al. [22] examined analytically the magnetohydrodynamic (MHD) viscous flow due to a shrinking sheet using the Adomian decomposition method (ADM) coupled with Padé approximants to handle the condition at infinity. Muhaimina et al. [23] studied the effect of the thermophoresis particle deposition on nonlinear MHD mixed convective heat and mass transfer over a porous shrinking sheet in the presence of suction.

The main objective of the present study is to find the solution for the problem of three-dimensional rotating flow induced by shrinking sheet with suction using the recently developed SHAM approach [24]. The problem was previously considered in [19] using the standard homotopy analysis method and in this work we use the new SHAM approach to 
solve the same problem. The SHAM method was shown to produce more improved results than the traditional HAM, see Motsa et al. [24]. In this work we compare the SHAM results for velocity distributions and shear stresses at the bounding walls against results generated using the very efficient MATLAB bvp4c in-built routine. The comparison indicates that there is excellent agreement between the two results proving that the SHAM is at least as good as the bvp4c and can be used in place of traditional numerical approaches such as RungeKutta methods, finite differences, Keller-Box method, for solving nonlinear boundary value problems.

\section{Mathematical Formulation}

We consider the steady, incompressible, three-dimensional flow of an electrically conducting viscous fluid between two horizontal parallel plates at $y=h$. Both the fluid and the plates rotate in unison with a constant angular velocity $\boldsymbol{\Omega}=\Omega j$, where $j$ is a unit vector in the $y$ direction. The plate $y=+h$ is rigid and stationary. The flow in the fluid system is caused due to shrinking of a porous plate at $y=-h$. The equations governing the rotating flow are Vajravelu and Kumar [17]:

$$
\begin{gathered}
\frac{\partial u}{\partial x}+\frac{\partial v}{\partial y}=0 \\
u \frac{\partial u}{\partial x}+v \frac{\partial u}{\partial y}+2 \Omega w=-\frac{1}{\rho} \frac{\partial p^{*}}{\partial x}+v\left[\frac{\partial^{2} u}{\partial x^{2}}+\frac{\partial^{2} u}{\partial y^{2}}\right]-\frac{\sigma B_{o}^{2}}{\rho} u \\
u \frac{\partial u}{\partial y}=-\frac{1}{\rho} \frac{\partial p^{*}}{\partial y}+v\left[\frac{\partial^{2} v}{\partial x^{2}}+\frac{\partial^{2} v}{\partial y^{2}}\right] \\
u \frac{\partial w}{\partial x}+v \frac{\partial w}{\partial y}-2 \Omega w=v\left[\frac{\partial^{2} w}{\partial x^{2}}+\frac{\partial^{2} w}{\partial y^{2}}\right]-\frac{\sigma B_{o}^{2}}{\rho} w
\end{gathered}
$$

The boundary conditions for the problem considered here are:

$$
\begin{gathered}
u=-a x, \quad v=-V, \quad w=0, \quad \text { at } y=-h, \\
u=0, \quad v=0, \quad w=0, \quad \text { at } y=+h,
\end{gathered}
$$

where $u, v$, and $w$ are the velocity components in $x-, y-$, and $z$-directions, respectively, $\rho$ is the density, $v$ is the kinematic viscosity, $\sigma$ is the electrical conductivity, $B_{o}$ is the magnetic induction, $p^{*}$ is the modified pressure, $a>0$ is the shrinking constant and $V>0$ is the suction velocity. In order to reduce (2.1)-(2.4) into a set of convenient ordinary differential equations, we introduce the similarity variable $\eta$ and the dimensionless variable $f$ and $g$ as follows:

$$
\eta=\frac{y}{h}, \quad u=-a x f^{\prime}(\eta), \quad v=\operatorname{ahf}(\eta), \quad w=\operatorname{axg}(\eta)
$$


The continuity equation (2.1) is automatically satisfied and (2.2)-(2.4), after eliminating the modified pressure, are characterized by the following model equations:

$$
\begin{gathered}
f^{i v}-M^{2} f^{\prime \prime}-2 K g^{\prime}-R\left(f^{\prime} f^{\prime \prime}-f f^{\prime \prime \prime}\right)=0, \\
g^{\prime \prime}-M^{2} g+2 K^{2} f^{\prime}-R\left(f^{\prime} g-f g^{\prime}\right)=0,
\end{gathered}
$$

where primes indicates differentiation with respect to $\eta$. In view of equation (2.6), the boundary equations (2.5), transform into:

$$
\begin{gathered}
f=1, \quad f^{\prime}=-1, \quad g=0, \quad \text { at } \eta=-1, \\
f=0, \quad f^{\prime}=0, \quad g=0, \quad \text { at } \eta=1,
\end{gathered}
$$

in which the suction parameter $\lambda$, the viscosity parameter $R$, the Hartman number $M$, and the rotating parameter $K^{2}$ are:

$$
\lambda=-\frac{V}{a h}, \quad R=\frac{a h^{2}}{v}, \quad M^{2}=\frac{\sigma B_{o}^{2} h^{2}}{\rho v}, \quad K^{2}=\frac{\Omega h^{2}}{v} .
$$

In the next section we will solve the nonlinear ordinary equation (2.7) by using the spectral homotopy analysis method (SHAM).

\section{Spectral Homotopy Analysis Method Solution}

In this section, we apply the SHAM approach to solve the governing equations (2.7)-(2.10). We begin by introducing the following transformation

$$
f(\eta)=f_{0}(\eta)+F(\eta), \quad g(\eta)=g_{0}(\eta)+G(\eta)
$$

where

$$
\begin{gathered}
f_{0}(\eta)=\left(\frac{\lambda}{2}-\frac{1}{4}\right)+\left(\frac{1-3 \lambda}{4}\right) \eta+\frac{\eta^{2}}{4}+\left(\frac{\lambda-1}{4}\right) \eta^{3} \\
g_{0}(\eta)=1-\eta^{2}
\end{gathered}
$$

are the initial approximations which are chosen to satisfy the boundary conditions (2.9)(2.10). Equation (3.1) is substituted into the governing equations (2.7)-(2.10) with the resulting equations written as a sum of their linear and nonlinear components as

$$
\begin{aligned}
& \mathcal{L}_{1}[F(\eta), G(\eta)]+\mathcal{N}_{1}[F(\eta), G(\eta)]=\Phi(\eta), \\
& \mathcal{L}_{2}[F(\eta), G(\eta)]+\mathcal{N}_{2}[F(\eta), G(\eta)]=\Psi(\eta),
\end{aligned}
$$


subject to the boundary conditions

$$
\begin{gathered}
F=0, \quad F^{\prime}=0, \quad G=0, \quad \text { at } \eta=-1, \\
F=0, \quad F^{\prime}=0, \quad G=0, \quad \text { at } \eta=1,
\end{gathered}
$$

where

$$
\begin{gathered}
\mathcal{L}_{1}[F(\eta), G(\eta)]=F^{i v}+a_{1} F^{\prime \prime \prime}+a_{2} F^{\prime \prime}+a_{3} F^{\prime}+a_{4} F-2 K^{2} G^{\prime}, \\
\mathcal{L}_{2}[F(\eta), G(\eta)]=G^{\prime \prime}+a_{1} G^{\prime}+a_{2} G+b_{1} F^{\prime}+b_{2} F, \\
\mathcal{N}_{1}[N(\eta), G(\eta)]=-R\left(F^{\prime} F^{\prime \prime}-F F^{\prime \prime \prime}\right), \\
\mathcal{N}_{2}[N(\eta), G(\eta)]=-R\left(F^{\prime} G-F G^{\prime}\right), \\
\Phi(\eta)=-\left[f_{0}^{i v}-M^{2} f_{0}^{\prime \prime}-2 K^{2} g_{0}^{\prime}-R\left(f_{0}^{\prime} f_{0}^{\prime \prime}-f_{0} f_{0}^{\prime \prime \prime}\right)\right], \\
\Psi(\eta)=-\left[g_{0}^{\prime \prime}-M^{2} g_{0}+2 K^{2} f_{0}^{\prime}-R\left(f_{0}^{\prime} g_{0}-f_{0} g_{0}^{\prime}\right)\right] .
\end{gathered}
$$

In the above definitions, the coefficient parameters are defined as

$$
\begin{gathered}
a_{1}=R_{0} f_{0}(\eta), \quad a_{2}=-M^{2}-R f_{0}^{\prime}(\eta), \quad a_{3}=-R f_{0}^{\prime \prime}, \\
a_{4}=R f_{0}^{\prime \prime \prime}, \quad b_{1}=2 K^{2}-R g_{0}, \quad b_{2}=R g_{0}^{\prime} .
\end{gathered}
$$

The SHAM approach builds on the basic ideas of the homotopy analysis method (HAM). However, for brevity, details of the HAM are omitted in this paper. For a detailed exposition of the HAM approach interested readers can refer to $[25,26]$ for a general description on the method and to [27-46] for the application of the HAM in boundary value problems over bounded domains. Thus, importing the ideas of the HAM approach, we construct the socalled zero-order deformation equations as

$$
\begin{aligned}
& (1-q) \mathcal{L}_{1}\left\{[\tilde{F}(\eta), \tilde{G}(\eta)]-\left[F_{0}(\eta), G_{0}(\eta)\right]\right\}=q \hbar\left\{\mathcal{L}_{1}[\tilde{F}(\eta), \tilde{G}(\eta)]+\mathcal{N}_{1}[\tilde{F}(\eta), \tilde{G}(\eta)]-\Phi(\eta)\right\}, \\
& (1-q) \mathcal{L}_{2}\left\{[\tilde{F}(\eta), \tilde{G}(\eta)]-\left[F_{0}(\eta), G_{0}(\eta)\right]\right\}=q \hbar\left\{\mathcal{L}_{2}[\tilde{F}(\eta), \tilde{G}(\eta)]+\mathcal{N}_{2}[\tilde{F}(\eta), \tilde{G}(\eta)]-\Psi(\eta)\right\},
\end{aligned}
$$

where $\hbar$ is the convergence controlling parameter, $q \in[0,1]$ is the embedding parameter, $\widetilde{F}(\eta)$ and $\tilde{G}(\eta)$ are unknown functions and $F_{0}(\eta)$ and $G_{0}(\eta)$ are initial approximations which are obtained as solutions of the linear part of equations (3.3)-(3.6) given as

$$
\begin{aligned}
& \mathcal{L}_{1}\left[F_{0}(\eta), G_{0}(\eta)\right]=\Phi(\eta), \\
& \left\llcorner_{2}\left[F_{0}(\eta), G_{0}(\eta)\right]=\Psi(\eta),\right.
\end{aligned}
$$


subject to the boundary conditions

$$
\begin{gathered}
F_{0}=0, \quad F_{0}^{\prime}=0, \quad G_{0}=0, \quad \text { at } \eta=-1, \\
F_{0}=0, \quad F_{0}^{\prime}=0, \quad G_{0}=0, \quad \text { at } \eta=1 .
\end{gathered}
$$

Following the HAM approach, the zero-order deformation deformation equations are differentiated $m$ times with respect to the embedding parameter $q$ then divided by $m$ ! with $q=0$ being set to the resulting equations to obtain the so-called higher-order deformation equations given by

$$
\begin{aligned}
& \mathcal{L}_{1}\left\{\left[F_{m}(\eta), G_{m}(\eta)\right]-x_{m}\left[F_{m-1}(\eta), G_{m-1}(\eta)\right]\right\}=\hbar R_{m, 1}, \\
& \mathcal{L}_{2}\left\{\left[F_{m}(\eta), G_{m}(\eta)\right]-x_{m}\left[F_{m-1}(\eta), G_{m-1}(\eta)\right]\right\}=\hbar R_{m, 2}
\end{aligned}
$$

subject to the boundary conditions

$$
F_{m}(-1)=F_{m}^{\prime}(-1)=F_{m}(1)=F^{\prime}(1)=G_{m}(-1)=G_{m}(1)=0,
$$

where

$$
\begin{gathered}
R_{m, 1}=F_{m-1}^{i v}+a_{1} F_{m-1}^{\prime \prime \prime}+a_{2} F_{m-1}^{\prime \prime}+a_{3} F_{m-1}^{\prime}+a_{4} F_{m}-2 K^{2} G_{m-1}^{\prime} \\
-R \sum_{n}^{=}\left(F_{n}^{\prime} F_{m-n-1}^{\prime \prime}-F_{n} F_{m-n-1}^{\prime \prime \prime}\right)-\left(1-\chi_{m}\right) \Phi(\eta), \\
R_{m, 2}=G_{m-1}^{\prime \prime}+a_{1} G_{m-1}^{\prime}+a_{2} G_{m-1}+b_{1} F_{m-1}^{\prime}+b_{2} F_{m} \\
-R \sum_{n}^{=}\left(F_{n}^{\prime} G_{m-n-1}-F_{n} G_{m-n-1}^{\prime}\right)-\left(1-\chi_{m}\right) \Psi(\eta), \\
x_{m}= \begin{cases}0, & m \leq 1, \\
1, & m>1 .\end{cases}
\end{gathered}
$$

We remark that, unlike in the standard HAM approach, the higher-order deformation equations (3.19)-(3.20) form a set of coupled ordinary differential equations (ODEs) instead of the decoupled set of ODEs that are generated in the HAM, that is in the SHAM approach the linear operators depend on both $F$ and $G$ (see (3.7) and (3.8)) whereas in the case of the HAM the linear operator would depend on one variable at a time. The SHAM technique also doe not depend on the rule of solution expression and the rule of ergodicity unlike the standard HAM. We use the Chebyshev pseudospectral method (see, e.g., $[47,48]$ ) to solve equations (3.19)-(3.20). The unknown functions $F_{m}(\eta)$ and $G_{m}(\eta)$ are approximated as truncated series of Chebyshev polynomials of the forms

$$
F_{m}(\eta) \approx \sum_{k}^{=} \widehat{F}_{m}\left(\eta_{k}\right) T_{k}\left(\eta_{j}\right), \quad G_{m}(\eta) \approx \sum_{k}^{=} \widehat{G}_{m}\left(\eta_{k}\right) T_{k}\left(\eta_{j}\right)
$$


where $j=0,1, \ldots, N, T_{k}$ is the $k$ th Chebyshev polynomial, and $\eta_{0}, \eta_{1}, \ldots, \eta_{N}$ are GaussLobatto collocation points (see [47]) defined by

$$
\eta_{j}=\cos \frac{\pi j}{N}, \quad j=0,1, \ldots, N
$$

Derivatives of the functions $F_{m}(\eta)$ and $G_{m}(\eta)$ at the collocation points are represented as

$$
\frac{d^{r} F_{m}}{d \eta^{r}}=\sum_{k}^{=} \Phi_{k j}^{r} F_{m}\left(\eta_{j}\right), \quad \frac{d^{r} G_{m}}{d \eta^{r}}=\sum_{k}^{=} \Phi_{k j}^{r} G_{m}\left(\eta_{j}\right)
$$

where $r$ is the order of differentiation and $\Phi$ is the Chebyshev spectral differentiation matrix $([47,48])$.

Substituting equations (3.22)-(3.24) in (3.19)-(3.20) yields

$$
\begin{aligned}
{\left[\begin{array}{ll}
A_{11} & A_{12} \\
A_{21} & A_{22}
\end{array}\right]\left[\begin{array}{l}
\mathbf{F}_{m} \\
\mathbf{G}_{m}
\end{array}\right]=} & \left(X_{m}+\hbar\right)\left[\begin{array}{ll}
A_{11} & A_{12} \\
A_{21} & A_{22}
\end{array}\right]\left[\begin{array}{l}
\mathbf{F}_{m-1} \\
\mathbf{G}_{m-1}
\end{array}\right] \\
& -\hbar\left(1-\chi_{m}\right)\left[\begin{array}{l}
\mathbf{\Phi} \\
\Psi
\end{array}\right]+\left[\begin{array}{l}
\mathbf{P}_{m-1} \\
\mathbf{Q}_{m-1}
\end{array}\right]
\end{aligned}
$$

subject to the boundary conditions

$$
\begin{array}{rlrl}
F_{m}\left(\eta_{N}\right)=0, \quad G_{m}\left(\eta_{N}\right) & =0, & & F_{m}\left(\eta_{0}\right)=0, \quad G_{m}\left(\eta_{0}\right)=0, \\
\sum_{k}^{=} \Phi_{N k} F_{m}\left(\eta_{k}\right) & =0, \quad & \sum_{k}^{=} \Phi_{0 k} F_{m}\left(\eta_{k}\right)=0,
\end{array}
$$

where

$$
\begin{aligned}
& A_{11}=\Phi^{4}+\mathbf{a}_{1} \Phi^{3}+\mathbf{a}_{2} \Phi^{2}+\mathbf{a}_{3} \boldsymbol{\Phi}+\mathbf{a}_{4} \\
& A_{12}=-2 K^{2} \boldsymbol{\oplus}, \quad a_{21}=\mathbf{b}_{1} \Phi+\mathbf{b}_{2} \\
& A_{22}=\Phi^{2}+\mathbf{a}_{1} \boldsymbol{\Phi}+\mathbf{a}_{2} \\
& \mathbf{F}_{m}=\left[F_{m}\left(\eta_{0}\right), F_{m}\left(\eta_{1}\right), \ldots, F_{m}\left(\eta_{N}\right)\right]^{T}, \quad \mathbf{G}_{m}=\left[G_{m}\left(\eta_{0}\right), G_{m}\left(\eta_{1}\right), \ldots, G_{m}\left(\eta_{N}\right)\right]^{T}, \\
& \boldsymbol{\Phi}=\left[\Phi\left(\eta_{0}\right), \Phi\left(\eta_{1}\right), \ldots, \Phi\left(\eta_{N}\right)\right]^{T}, \quad \Psi=\left[\Psi\left(\eta_{0}\right), \Psi\left(\eta_{1}\right), \ldots, \Psi\left(\eta_{N}\right)\right]^{T}, \\
& \mathbf{P}_{m-1}=-R \sum_{n}^{=}\left(\boldsymbol{\Phi} F_{n} \boldsymbol{\Phi}^{2} F_{m-1-n}-F_{n} \Phi^{3} F_{m-1-n}\right), \\
& \mathbf{Q}_{m-1}=-R \sum_{n}^{=}\left(\Phi F_{n} G_{m-1-n}-F_{n} \oplus G_{m-1-n}\right) .
\end{aligned}
$$


Table 1: Comparison of the values of wall shear stresses $f^{\prime \prime}(-1), g^{\prime}(-1)$ with the numerical solution for different orders of the SHAM approximation when $\lambda$ is varied with $\hbar=-1, M=0.5, K=0.5$, and $R=0.2$.

\begin{tabular}{lcccccc}
\hline$\lambda$ & \multicolumn{2}{c}{$f^{\prime \prime}(-1)$} & & \multicolumn{3}{c}{$g^{\prime}(-1)$} \\
2nd order & Numerical & 1st-order & 2nd order & Numerical \\
\hline 0.0 & 2.036755 & 2.036755 & 2.036755 & -0.086956 & -0.086956 & -0.086956 \\
0.5 & 1.290768 & 1.290768 & 1.290768 & -0.204070 & -0.204070 & -0.204070 \\
1.0 & 0.517318 & 0.517318 & 0.517318 & -0.337831 & -0.337831 & -0.337831 \\
1.5 & -0.285247 & -0.285247 & -0.285247 & -0.490317 & -0.490317 & -0.490317 \\
2.0 & -1.118646 & -1.118646 & -1.118646 & -0.663878 & -0.663879 & -0.663879 \\
2.5 & -1.984669 & -1.984669 & -1.984669 & -0.861178 & -0.861182 & -0.861182 \\
3.0 & -2.885172 & -2.885172 & -2.885172 & -1.085248 & -1.085259 & -1.085259 \\
3.5 & -3.822080 & -3.822080 & -3.822080 & -1.339543 & -1.339572 & -1.339572 \\
4.0 & -4.797383 & -4.797383 & -4.797383 & -1.628018 & -1.628084 & -1.628084 \\
\hline
\end{tabular}

Table 2: Comparison of the values of wall shear stresses $f^{\prime \prime}(-1), g^{\prime}(-1)$ with the numerical solution for different orders of the SHAM approximation when $M$ is varied with $\hbar=-1, \lambda=0.5, K=0.5$, and $R=0.5$.

\begin{tabular}{ccccccc}
\hline$M$ & \multicolumn{2}{c}{$f^{\prime \prime}(-1)$} & & $g^{\prime}(-1)$ \\
2nd order & Numerical & 1st-order & 2nd order & Numerical \\
\hline 0.0 & 1.214539 & 1.214539 & 1.214539 & -0.226272 & -0.226273 & -0.226273 \\
0.5 & 1.271125 & 1.271125 & 1.271125 & -0.213930 & -0.213930 & -0.213930 \\
1.0 & 1.431238 & 1.431237 & 1.431237 & -0.186192 & -0.186200 & -0.186200 \\
1.5 & 1.671689 & 1.671687 & 1.671687 & -0.156767 & -0.156789 & -0.156789 \\
2.0 & 1.966684 & 1.966678 & 1.966678 & -0.131647 & -0.131681 & -0.131681 \\
2.5 & 2.295272 & 2.295262 & 2.295262 & -0.111685 & -0.111723 & -0.111723 \\
3.0 & 2.643325 & 2.643310 & 2.643310 & -0.096121 & -0.096157 & -0.096157 \\
3.5 & 3.002340 & 3.002322 & 3.002322 & -0.083955 & -0.083986 & -0.083986 \\
4.0 & 3.367516 & 3.367496 & 3.367496 & -0.074329 & -0.074355 & -0.074355 \\
\hline
\end{tabular}

In the above definitions the superscript $T$ denotes transpose, and $\mathbf{a}_{i}, \mathbf{b}_{i}$ denotes diagonal matrices, $\mathbf{I}$ is an identity matrix of size $(N+1) \times(N+1)$. The boundary conditions (3.25) and (3.27) are imposed on equation (3.24) are the resulting equation is solved for $\mathbf{F}_{m}$ and $\mathbf{G}_{m}$ iteratively using $F_{0}(\eta)$ and $G_{0}(\eta)$, which are obtained as solutions of (3.15)-(3.18), as a starting point.

\section{Results and Discussion}

In this section we give the SHAM results for the four main parameters affecting the flow. We remark that, all the SHAM results presented in this work were obtained using $N=50$ collocation points. Tables $1-4$ give a comparison of the SHAM results for $f^{\prime \prime}(-1), f^{\prime \prime}(1), g^{\prime}(-1)$ and $g^{\prime}(1)$ at different orders of approximation against the numerical results. The numerical results are obtained using the MATLAB routine bvp4c. Table 1 shows that full convergence of the SHAM is achieved by as early as the second-order, substantiating the claim that SHAM is a very powerful technique. We observe that convergence is achieved at second-order of approximation for all parameter values or combinations of these parameters as depicted in all these tables. We observe in Table 1 that the suction parameter $\lambda$ significantly affects the shear stress exerted by the shrinking sheet at $\eta=-1$. Increasing the values of $\lambda$ causes much 


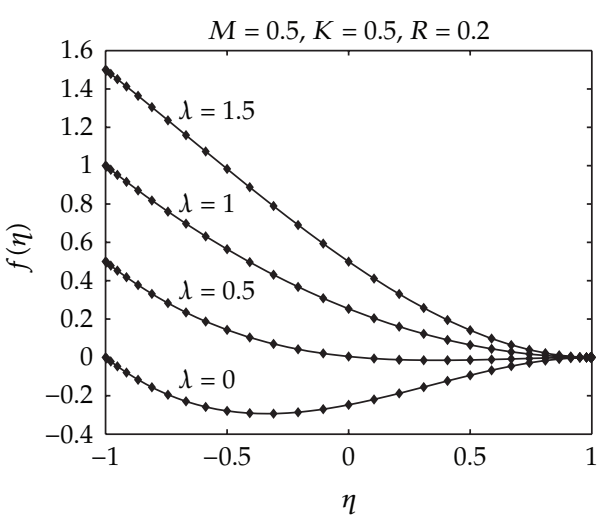

(a)

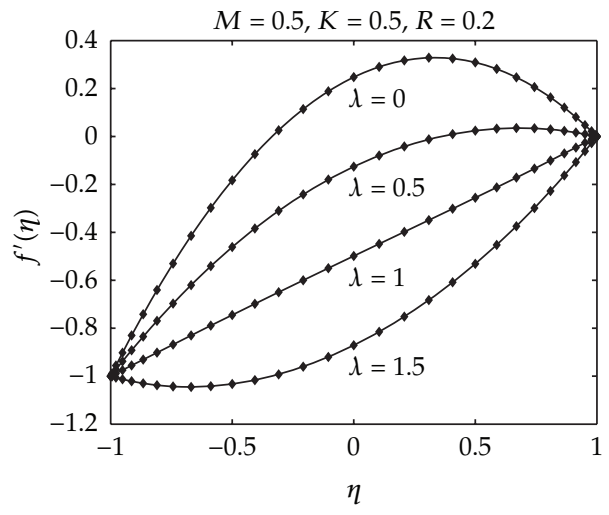

(b)

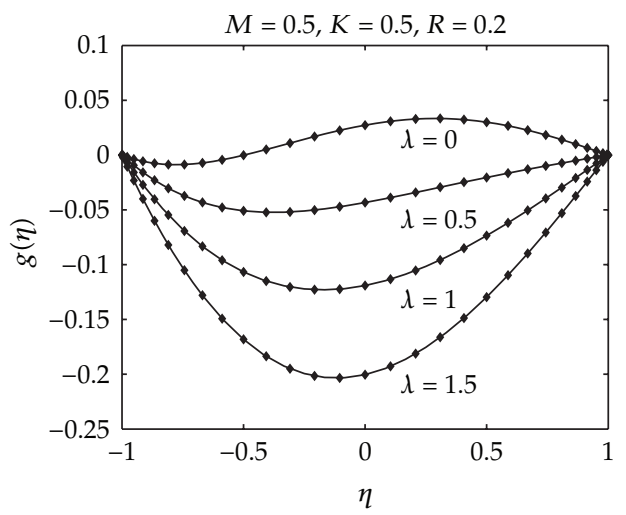

(c)

Figure 1: Influence of the suction parameter $\lambda$ on $f(\eta), f^{\prime}(\eta)$ and $g(\eta)$, when $\hbar=-1$. Numerical solution (solid line) is compared against the SHAM 1st-order approximation (diamonds).

reductions in the shear stress at $\eta=-1$ as shown by both values of $f^{\prime \prime}(-1)$ and $g^{\prime}(-1)$. This is because blowing gives rise to a thicker velocity boundary layer, thereby causing a decrease in the velocity gradient at the surface.

From Table 2, it is observed that the Hartman number tends to greatly increase the local skin friction at the shrinking sheet $(\eta=-1)$. This is because the increase in the magnetic field strength leads to a thinner velocity boundary layer, thereby causing an increase in the velocity gradient at the wall. In Table 3 we observe the influence of the rotation parameter $K$ on the shear stress $f^{\prime \prime}(-1)$ and $g^{\prime}(-1)$. We observe that both $f^{\prime \prime}(-1)$ and $g^{\prime}(-1)$ decrease as the values of $K$ increase. In Table 4 we observe that $f^{\prime \prime}(-1)$ decreases by increasing $R$ and $g^{\prime}(-1)$ increases as $R$ increases.

Figures 1-4 have been plotted to depict the influence of suction parameter $\lambda$, the Hartman number $M$, rotation parameter $K$ and viscosity parameter $R$. On these figures, we also give comparisons between the numerical results and the second-order SHAM solutions and excellent agreement between the two sets of results was always achieved. In Figure 1, we have the effects of varying the values of suction parameter $\lambda$ on $f, f^{\prime}$ and $g$. From Figure 1 it is found that $f$ increases as $\lambda$ increases and $f$ has maximum values at the lower end of the plate (shrinking sheet). It is clearly depicted in Figure 1 that $f^{\prime}$ decreases when values of 


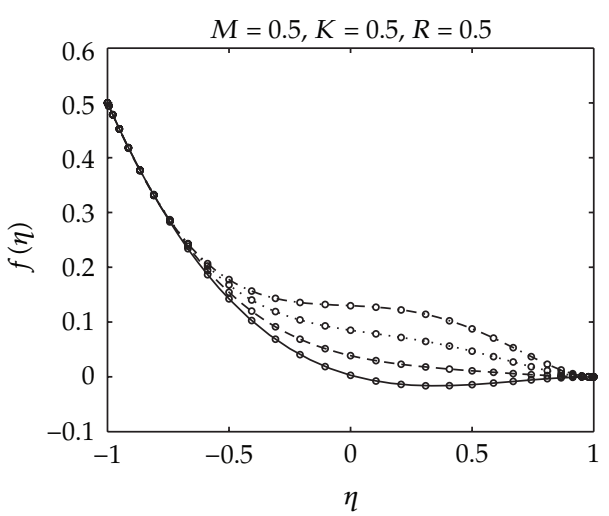

(a)

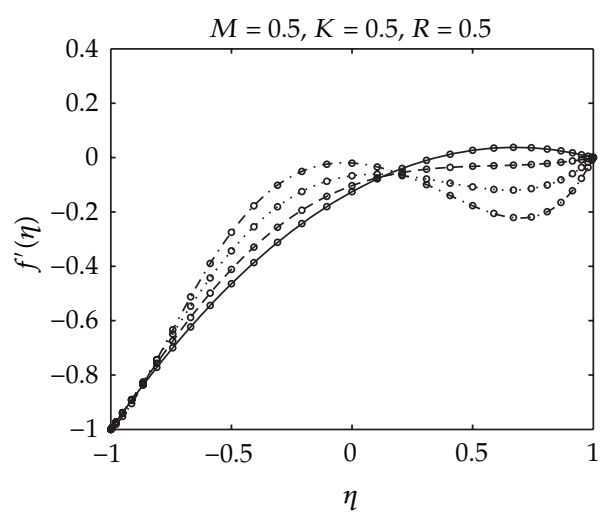

(b)

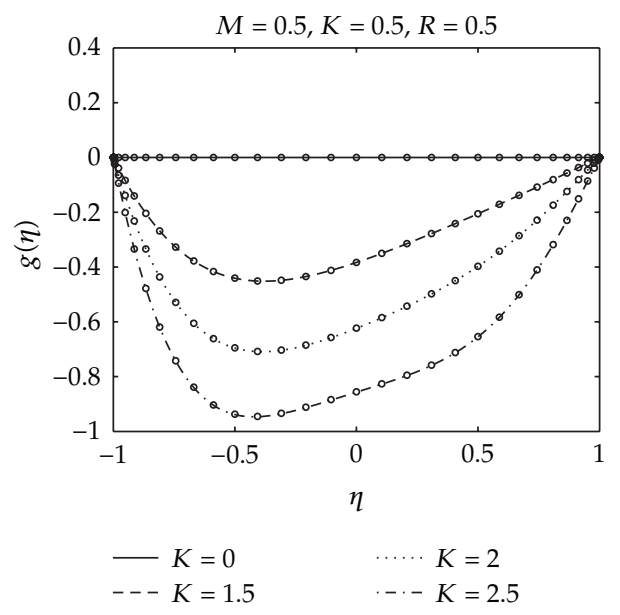

(c)

Figure 2: Influence of $K$ on $f(\eta), f^{\prime}(\eta)$ and $g(\eta)$, when $\hbar=-1$. Numerical solution (lines) is compared against the SHAM 1st-order approximation (open circles).

Table 3: Comparison of the values of wall shear stresses $f^{\prime \prime}(-1), g^{\prime}(-1)$ with the numerical solution for different orders of the SHAM approximation when $K$ is varied with $\hbar=-1, \lambda=0.5, R=0.2$, and $M=0.5$.

\begin{tabular}{ccccccc}
\hline$K$ & \multicolumn{3}{c}{$f^{\prime \prime}(-1)$} & & $g^{\prime}(-1)$ \\
4th order & Numerical & 2nd order & 4th order & Numerical \\
\hline 0.0 & 1.290778 & 1.290778 & 1.290778 & -0.000000 & -0.000000 & -0.000000 \\
0.5 & 1.290768 & 1.290768 & 1.290768 & -0.204070 & -0.204070 & -0.204070 \\
1.0 & 1.289629 & 1.289629 & 1.289629 & -0.808721 & -0.808721 & -0.808721 \\
1.5 & 1.267876 & 1.267876 & 1.267876 & -1.758288 & -1.758288 & -1.758288 \\
2.0 & 1.138135 & 1.138137 & 1.138137 & -2.939868 & -2.939865 & -2.939865 \\
2.5 & 0.783860 & 0.783869 & 0.783869 & -4.302754 & -4.302744 & -4.302744 \\
3.0 & 0.176755 & 0.176771 & 0.176771 & -5.885655 & -5.885639 & -5.885639 \\
3.5 & -0.664164 & -0.664146 & -0.664146 & -7.721068 & -7.721051 & -7.721051 \\
4.0 & -1.740066 & -1.740049 & -1.740049 & -9.812337 & -9.812322 & -9.812322 \\
\hline
\end{tabular}




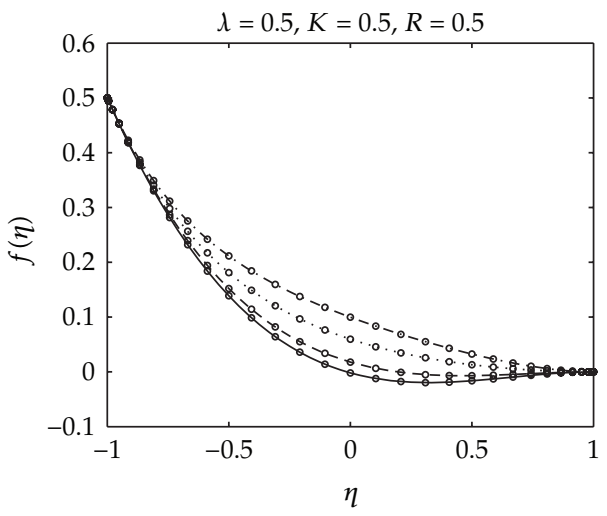

(a)

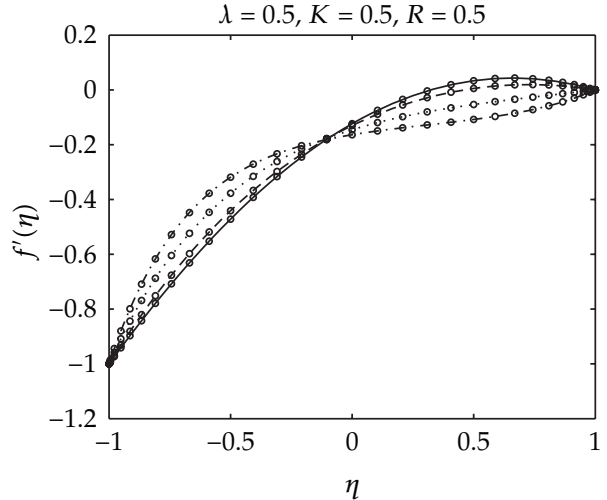

(b)

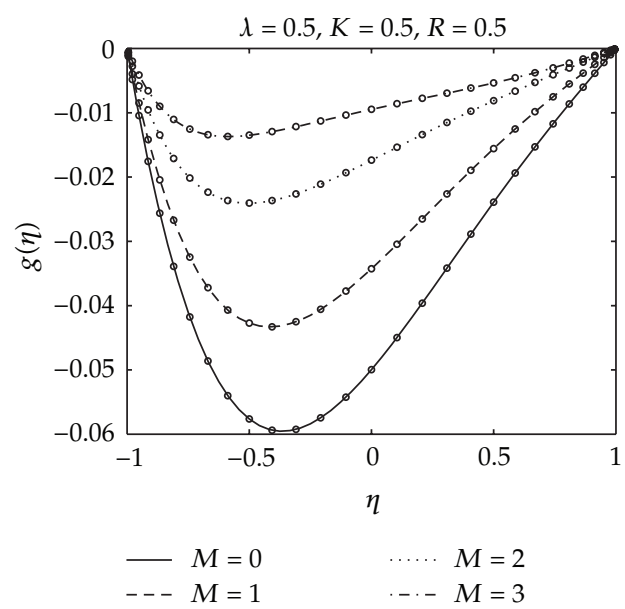

(c)

Figure 3: Influence of $M$ on $f(\eta), f^{\prime}(\eta)$ and $g(\eta)$, when $\hbar=-1$. Numerical solution (lines) is compared against the SHAM 1st-order approximation (open circles).

Table 4: Comparison of the values of wall shear stresses $f^{\prime \prime}(-1), g^{\prime}(-1)$ with the numerical solution for different orders of the SHAM approximation when $R$ is varied with $\hbar=-0.98, \lambda=0.5, K=0.2$, and $M=0.5$.

\begin{tabular}{ccccccc}
\hline$R$ & \multicolumn{2}{c}{$f^{\prime \prime}(-1)$} & & $g^{\prime}(-1)$ & \\
& 2nd order & 4th order & Numerical & 2nd order & 4th order & Numerical \\
\hline 0.0 & 1.303332 & 1.303332 & 1.303332 & -0.197931 & -0.197931 & -0.197931 \\
0.2 & 1.290768 & 1.290768 & 1.290768 & -0.204070 & -0.204070 & -0.204070 \\
0.4 & 1.277782 & 1.277782 & 1.277782 & -0.210552 & -0.210552 & -0.210552 \\
0.6 & 1.264354 & 1.264354 & 1.264354 & -0.217406 & -0.217406 & -0.217406 \\
0.8 & 1.250463 & 1.250463 & 1.250463 & -0.224665 & -0.224665 & -0.224665 \\
1.0 & 1.236085 & 1.236085 & 1.236085 & -0.232365 & -0.232365 & -0.232365 \\
2.0 & 1.155958 & 1.155958 & 1.155958 & -0.279118 & -0.279118 & -0.279118 \\
3.0 & 1.059078 & 1.059078 & 1.059078 & -0.346195 & -0.346202 & -0.346202 \\
\hline
\end{tabular}




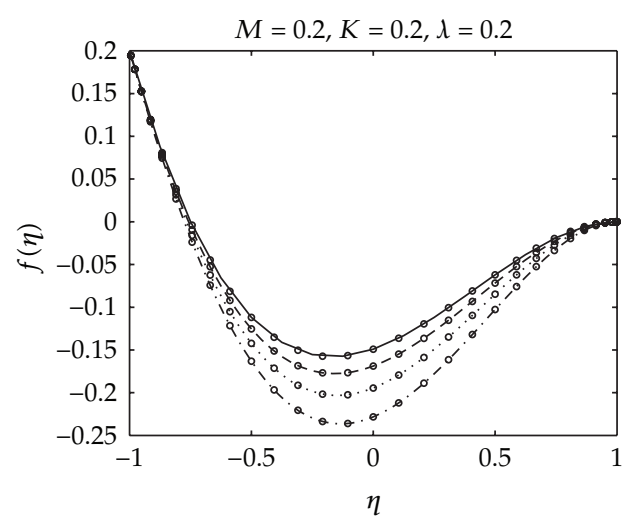

(a)

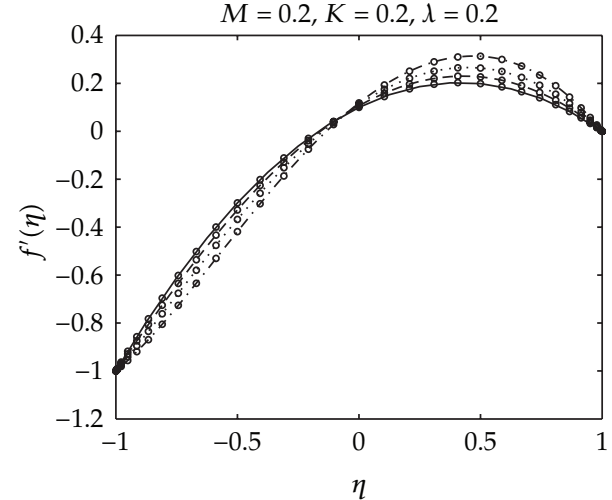

(b)

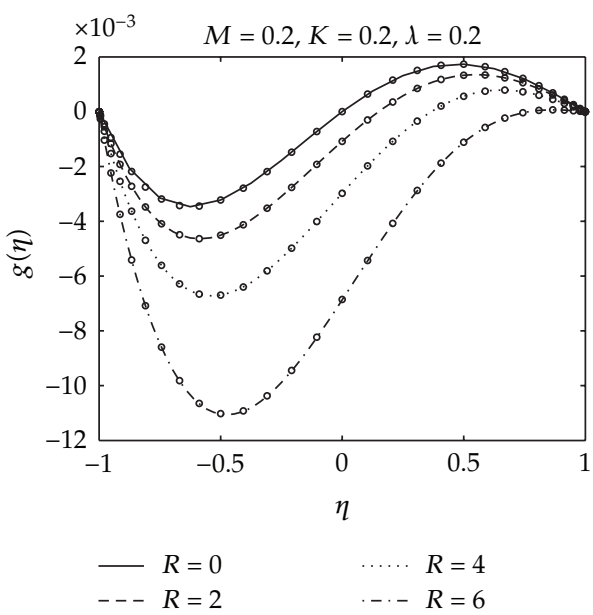

(c)

Figure 4: Influence of $R$ on $f(\eta), f^{\prime}(\eta)$ and $g(\eta)$, when $\hbar=-1$. Numerical solution (lines) is compared against the SHAM 1st-order approximation (open circles).

the suction parameter increase. It is also observed that for small values of $\lambda, f^{\prime}$ has large values near the center of the channel. Figure 1 also elucidates the effects of $\lambda$ on $g$. We observe that $g$ decreases as $\lambda$ increases and the decrease is more pronounced at the center of the channel as compared to near the plates.

In Figure 2 we depict the effects of the rotation parameter $K$ on $f, f^{\prime}$ and $g$. We observe in this figure that near the shrinking plate, the rotation parameter has no effect on $f$. However, as we move towards the center of the channel $f$ increases as $K$ increases. Figure 2 indicates that $f^{\prime}$ increases near the shrinking sheet and also that the boundary layer thickness decreases near this sheet. As we approach the nonpermeable plate, we observe that $f^{\prime}$ is now a decreasing function of $K$. In this figure we observe that $f^{\prime}$ is not a monotonous function of $K$. We also have the effects of $K$ on $g$ depicted in Figure 2. We clearly see that $g$ decreases as $K$ increases.

Figure 3 depicts the effects of $M$ on $f, f^{\prime}$, and $g$. We observe in this figure that $f$ is an increasing function of the Hartman number $M$. It is observed in Figure 3 that $f^{\prime}$ initially increases but then decreases after the center of the channel as values of $M$ increase. 
The Hartman number $M$ significantly reduces the values of $g$. We observe in this Figure 3 that $g$ has quite opposite behaviour when compared with the suction parameter $\lambda$.

Lastly, in Figure 4 we show the effects of viscosity parameter $R$ on $f, f^{\prime}$ and $g$. We observe that viscosity reduces the velocity $f$ and increases the boundary layer thickness. The minimum values of $f$ are observed near the center of the channel. It is noted in Figure 4 that increasing the values of $R$ initially decreases $f^{\prime}$ but increases it after the channel center. It can also be observed in Figure 4 that $R$ significantly affects $g$. As $R$ increases, $g$ values are greatly reduced attaining their minimum values near the shrinking sheet.

\section{Conclusion}

The three-dimensional rotating flow in a channel generated by a shrinking sheet is studied. The spectral-homotopy analysis method is used to solve the nonlinear system of ordinary differential equations. The variations of the four main parameters on the velocity $\left(f, f^{\prime}, g\right)$ and wall shear stress $\left(f^{\prime \prime}(-1),-g^{\prime}(-1)\right)$ are discussed through graphs and tables, respectively. The following observations have been made.

(i) The SHAM rapidly converges to the numerical results generated by MATLAB bvp4c routine.

(ii) The velocity $f$ increases for $\lambda, M$, and $K$ but decreases for $R$.

(iii) The velocity $f^{\prime}$ decreases for increasing values of $\lambda$ but is not a monotonous function of $K, M$, and $R$.

(iv) The velocity $g$ decreases for increasing values of $\lambda, K$ and $R$ but increases for increasing values of $M$.

\section{Acknowledgment}

The authors wish to acknowledge financial support from the University of Swaziland.

\section{References}

[1] B. C. Sakiadis, "Boundary-layer behaviour on continuous solid surfaces," AIChE, vol. 7, p. $268,1961$.

[2] L. J. Crane, "Flow past a stretching plate," Zeitschrift für angewandte Mathematik und Physik, vol. 21, pp. $445-447,1970$.

[3] E. Magyari and B. Keller, "Heat and mass transfer in the boundary layers on an exponentially stretching continuous surface," Journal of Physics D, vol. 32, no. 5, pp. 577-585, 1999.

[4] E. Magyari and B. Keller, "Exact solutions for self-similar boundary-layer flows induced by permeable stretching walls," European Journal of Mechanics. B, vol. 19, no. 1, pp. 109-122, 2000.

[5] E. Magyari and B. Keller, "A direct method to calculate the heat transfer coefficient of steady similar boundary layer flows induced by continuous moving surface," International Journal of Thermal Sciences, vol. 44, pp. 245-254, 2005.

[6] S.-J. Liao and I. Pop, "Explicit analytic solution for similarity boundary layer equations," International Journal of Heat and Mass Transfer, vol. 47, no. 1, pp. 75-85, 2004.

[7] E. M. Sparrow and J. P. Abraham, "Universal solutions for the streamwise variation of the temperature of a moving sheet in the presence of a moving fluid," International Journal of Heat and Mass Transfer, vol. 48, no. 15, pp. 3047-3056, 2005.

[8] J. P. Abraham and W. M. Sparrow, "Friction drag resulting from the simultaneous imposed motions of a freestream and its bounding surface," International Journal of Heat and Fluid Flow, vol. 26, pp. 289-295, 2005. 
[9] C. Y. Wang, "Stretching a surface in a rotating fluid," Journal of Applied Mathematics and Physics, vol. 39, pp. 177-185, 1999.

[10] V. Rajeswari and G. Nath, "Unsteady flow over a stretching surface in a rotating fluid," International Journal of Engineering Science, vol. 30, no. 6, pp. 747-756, 1992.

[11] R. Nazar, N. Amin, and I. Pop, “Unsteady boundary layer flow due to a stretching surface in a rotating fluid," Mechanics Research Communications, vol. 31, pp. 121-128, 2004.

[12] P. D. Ariel, "On computation of the three-dimensional flow past a stretching sheet," Applied Mathematics and Computation, vol. 188, no. 2, pp. 1244-1250, 2007.

[13] M. M. Rashidi and S. Dinarvand, "Purely analytic approximate solutions for steady three-dimensional problem of condensation film on inclined rotating disk by homotopy analysis method," Nonlinear Analysis. Real World Applications, vol. 10, no. 4, pp. 2346-2356, 2009.

[14] M. Kumari and G. Nath, "Unsteady MHD mixed convection flow over an impulsively stretched permeable vertical surface in a quiescent fluid," International Journal of Non-Linear Mechanics, vol. 45, no. 3, pp. 310-319, 2010.

[15] T. Fang, J. Zhang, and S. Yao, "Slip MHD viscous flow over a stretching sheet-an exact solution," Communications in Nonlinear Science and Numerical Simulation, vol. 14, no. 11, pp. 3731-3737, 2009.

[16] H. S. Takhar, A. J. Chamkha, and G. Nath, "MHD flow over a moving plate in a rotating fluid with magnetic field, Hall currents and free stream velocity," International Journal of Engineering Science, vol. 40, pp. 1511-1527, 2002.

[17] K. Vajravelu and B. V. R. Kumar, "Analytical and numerical solutions of a coupled non-linear system arising in a three-dimensional rotating flow," International Journal of Non-Linear Mechanics, vol. 39, no. 1, pp. 13-24, 2004.

[18] C. Y. Wang, "Liquid film on an unsteady stretching surface," Quarterly of Applied Mathematics, vol. 48, no. 4, pp. 601-610, 1990.

[19] T. Hayat, M. Sajid, and I. Pop, “Three-dimensional flow over a stretching surface in a viscoelastic fluid," Nonlinear Analysis. Real World Applications, vol. 9, no. 4, pp. 1811-1822, 2008.

[20] M. Sajid and T. Hayat, "The application of homotopy analysis method for MHD viscous flow due to a shrinking sheet," Chaos, Solitons and Fractals, vol. 39, no. 3, pp. 1317-1323, 2009.

[21] B. Yao and J. Chen, "A new analytical solution branch for the Blasius equation with a shrinking sheet," Applied Mathematics and Computation, vol. 215, no. 3, pp. 1146-1153, 2009.

[22] N. F. M. Noor, A. S. Kechil, and I. Hashim, "Simple non-perturbative solution for MHD viscous flow due to a shrinking sheet," Communications in Nonlinear Science and Numerical Simulation, vol. 15, no. 2, pp. 144-148, 2010.

[23] Muhaimina, R. Kandasamya, and I. Hashim, "Effect of chemical reaction, heat and mass transfer on nonlinear boundary layer past a porous shrinking sheet in the presence of suction," Nuclear Engineering and Design, vol. 240, no. 5, pp. 933-939, 2010.

[24] S. S. Motsa, P. Sibanda, and S. Shateyi, "A new spectral-homotopy analysis method for solving a nonlinear second order BVP," Communications in Nonlinear Science and Numerical Simulation, vol. 15, no. 9, pp. 2293-2302, 2010.

[25] S. Liao, "Notes on the homotopy analysis method: some definitions and theorems," Communications in Nonlinear Science and Numerical Simulation, vol. 14, no. 4, pp. 983-997, 2009.

[26] S. Liao, Beyond Perturbation, vol. 2 of CRC Series: Modern Mechanics and Mathematics, Chapman \& Hall/CRC, Boca Raton, Fla, USA, 2004.

[27] A. S. Bataineh, M. S. M. Noorani, and I. Hashim, "Modified homotopy analysis method for solving systems of second-order BVPs," Communications in Nonlinear Science and Numerical Simulation, vol. 14, no. 2, pp. 430-442, 2009.

[28] A. Sami Bataineh, M. S. M. Noorani, and I. Hashim, "On a new reliable modification of homotopy analysis method," Communications in Nonlinear Science and Numerical Simulation, vol. 14, no. 2, pp. 409-423, 2009.

[29] S. Dinarvand and M. M. Rashidi, "A reliable treatment of a homotopy analysis method for twodimensional viscous flow in a rectangular domain bounded by two moving porous walls," Nonlinear Analysis. Real World Applications, vol. 11, no. 3, pp. 1502-1512, 2010.

[30] G. Domairry, A. Mohsenzadeh, and M. Famouri, "The application of homotopy analysis method to solve nonlinear differential equation governing Jeffery-Hamel flow," Communications in Nonlinear Science and Numerical Simulation, vol. 14, no. 1, pp. 85-95, 2009.

[31] T. Hayat, T. Javed, and M. Sajid, "Analytic solution for rotating flow and heat transfer analysis of a third-grade fluid," Acta Mechanica, vol. 191, no. 3-4, pp. 219-229, 2007. 
[32] T. Hayat, S. B. Khan, M. Sajid, and S. Asghar, "Rotating flow of a third grade fluid in a porous space with Hall current," Nonlinear Dynamics, vol. 49, no. 1-2, pp. 83-91, 2007.

[33] T. Hayat and M. Sajid, "On analytic solution for thin film flow of a forth grade fluid down a vertical cylinder.," Physics Letters A, vol. 361, pp. 316-322, 2007.

[34] T. Hayat and M. Sajid, "Analytic solution for axisymmetric flow and heat transfer of a second grade fluid past a stretching sheet," International Journal of Heat and Mass Transfer, vol. 50, no. 1-2, pp. 75-84, 2007.

[35] T. Hayat, Z. Abbas, M. Sajid, and S. Asghar, "The influence of thermal radiation on MHD flow of a second grade fluid," International Journal of Heat and Mass Transfer, vol. 50, no. 5-6, pp. 931-941, 2007.

[36] T. Hayat and M. Sajid, "Homotopy analysis of MHD boundary layer flow of an upper-convected Maxwell fluid," International Journal of Engineering Science, vol. 45, pp. 393-401, 2007.

[37] T. Hayat, N. Ahmed, M. Sajid, and S. Asghar, “On the MHD flow of a second grade fluid in a porous channel," Computers \& Mathematics with Applications, vol. 54, no. 3, pp. 407-414, 2007.

[38] T. Hayat, M. Khan, and M. Ayub, "The effect of the slip condition on flows of an Oldroyd 6-constant fluid," Journal of Computational and Applied Mathematics, vol. 202, no. 2, pp. 402-413, 2007.

[39] T. Hayat, N. Ahmed, and M. Sajid, "Analytic solution for MHD flow of a third order fluid in a porous channel," Journal of Computational and Applied Mathematics, vol. 214, no. 2, pp. 572-582, 2008.

[40] T. Hayat, M. Khan, and M. Ayub, "On the explicit analytic solutions of an Oldroyd 6-constant fluid," International Journal of Engineering Science, vol. 42, no. 2, pp. 123-135, 2004.

[41] T. Hayat, R. Naz, and M. Sajid, "On the homotopy solution for Poiseuille flow of a fourth grade fluid," Communications in Nonlinear Science and Numerical Simulation, vol. 15, no. 3, pp. 581-589, 2010.

[42] T. Hayat, R. Ellahi, P. D. Ariel, and S. Asghar, "Homotopy solution for the channel flow of a third grade fluid," Nonlinear Dynamics, vol. 45, no. 1-2, pp. 55-64, 2006.

[43] J. Hosseini and A. F. Mohammad, "Application of homotopy analysis method for nonlinear SturmLiouville problems," Journal of Advanced Research in Differential Equations, vol. 1, pp. 11-20, 2009.

[44] A. Mehmood and A. Ali, "Heat transfer analysis of three-dimensional flow in a channel of lower stretching wall," Journal of the Taiwan Institute of Chemical Engineers, vol. 41, no. 1, pp. 29-34, 2010.

[45] M. M. Rashidi and S. Dinarvand, "Purely analytic approximate solutions for steady three-dimensional problem of condensation film on inclined rotating disk by homotopy analysis method," Nonlinear Analysis. Real World Applications, vol. 10, no. 4, pp. 2346-2356, 2009.

[46] M. Sajid, T. Hayat, S. Aghar, and K. Varjravelu, "Analytic solution for axisymetric flow over a nonlinear stretching sheet," Archives of Applied Mechanics, vol. 78, pp. 127-134, 2008.

[47] C. Canuto, M. Y. Hussaini, A. Quarteroni, and T. A. Zang, Spectral Methods in Fluid Dynamics, Springer Series in Computational Physics, Springer, New York, NY, USA, 1988.

[48] W. S. Don and A. Solomonoff, "Accuracy and speed in computing the Chebyshev collocation derivative," SIAM Journal on Scientific Computing, vol. 16, no. 6, pp. 1253-1268, 1995. 


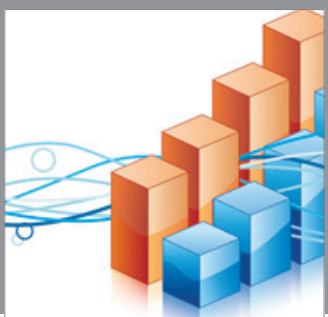

Advances in

Operations Research

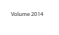

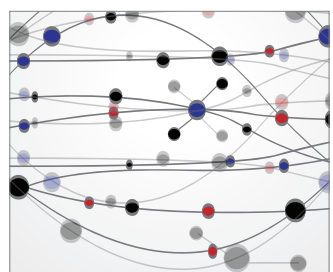

\section{The Scientific} World Journal
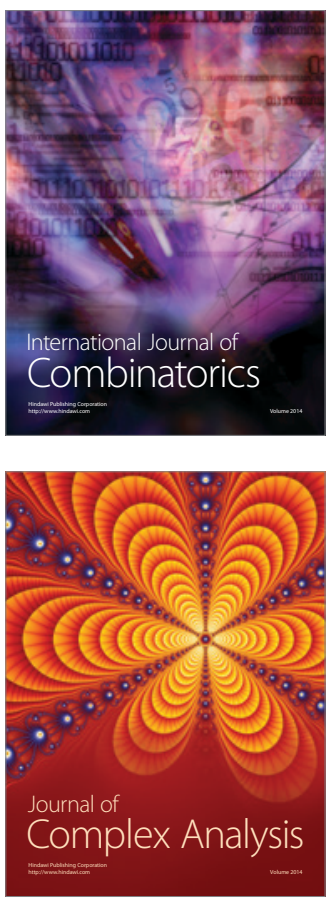

International Journal of

Mathematics and

Mathematical

Sciences
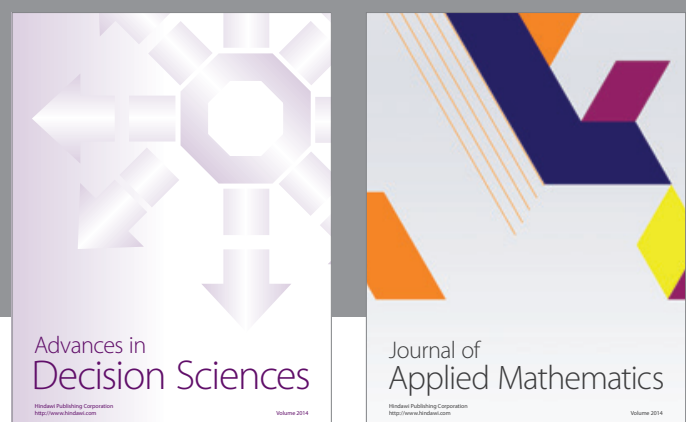

Journal of

Applied Mathematics
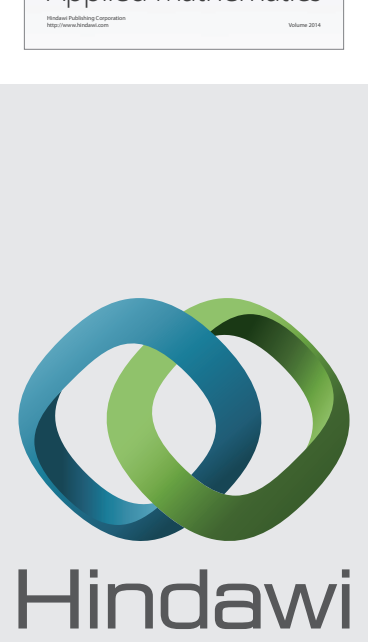

Submit your manuscripts at http://www.hindawi.com
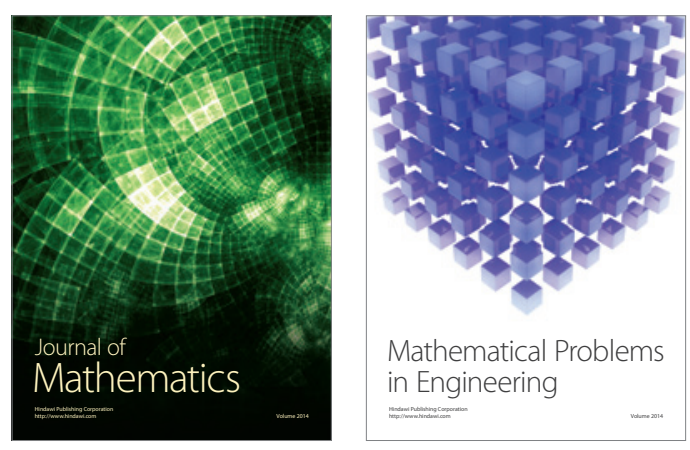

Mathematical Problems in Engineering
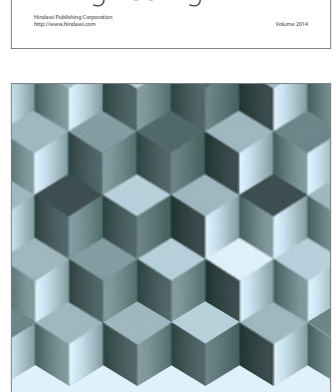

Journal of

Function Spaces
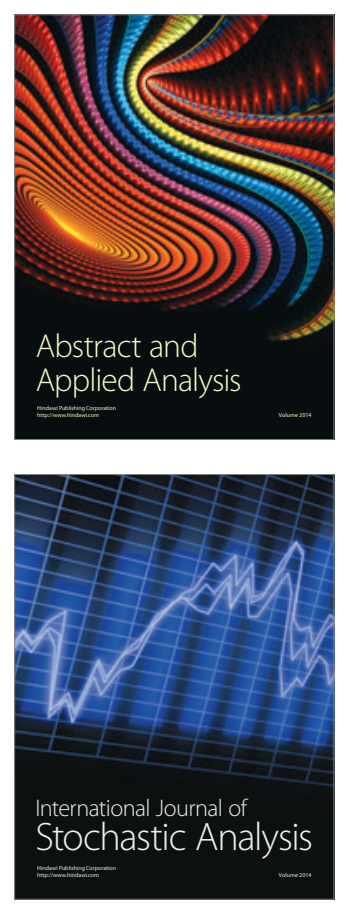

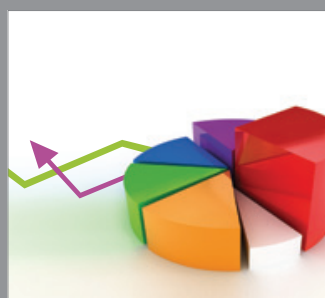

ournal of

Probability and Statistics

Promensencen
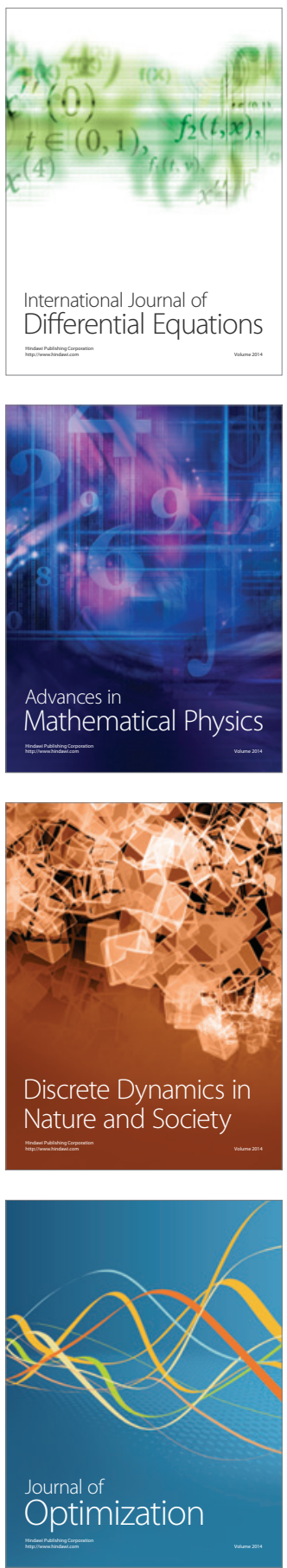